\title{
EVALUAR... PARA QUE APRENDAN MÁS
}

Danilo A. Quiñones Reyna

Universidad Pedagógica "Pepito Tey", Cuba

\section{INTRODUCCIÓN}

La evaluación es un tema muy recurrente en todos los ámbitos sociales: en la familia se polemiza acerca de cómo se debió hacer esto o lo otro por cada uno de sus miembros, o si lo que se hizo es o no correcto, sobre todo cuando se trata de niños, adolescentes y jóvenes que son los que más orientaciones necesitan como complemento de un proceso formativo que a partir de la edad escolar regenta la escuela. Es un tema omnipresente, está de moda. No solo por el hecho de evaluar, sino, además, porque frecuentemente se habla y escribe sobre este particular.

Todos, de una forma u otra queremos adoptar una posición ante los problemas de la vida social, cada persona quiere identificarse de acuerdo con sus intereses, particularidades personales, intenciones y propósitos. Así, educandos y aprendices reclaman el espacio para el despliegue de su identidad en la relación con los otros, mutuamente hacen valoraciones del proceso por el que han transitado y de los resultados alcanzados.

La autovaloración y autorregulación, como principios de la metacognición, evolucionan hacia un estado configuracional que revela las necesidades que tiene cada sujeto de demostrar lo que va aprendiendo en un proceso de constante transformación de sus modos de actuación.

Enfrentar o dirigir el proceso evaluativo de los alumnos es un elemento de complicación, porque desde la perspectiva del maestro; en la generalidad de los casos, piensan que lo más importante es que todos sus alumnos estén evaluados en una etapa o período del curso escolar, por otra parte, es una tendencia muy manida el advertir sobre las ventajas y desventajas de la evaluación desde posiciones de un discurso académico y pensar que así llegaríamos a solucionar los problemas que más nos afectan al respecto. Es por ello que consideramos importante que los maestros de la escuela primaria reflexionen a partir de las siguientes interrogantes:

- ¿Saben nuestros maestros implementar las funciones de la evaluación en la dirección del proceso de enseñanza-aprendizaje, sin que esto se convierta en un momento de tensión para los sujetos que aprenden?

- ¿Quién se beneficia con la evaluación?

- ¿Al servicio de quién se pone?

- ¿Qué es evaluar en la escuela primaria?

- ¿Están preparados los maestros para dar un seguimiento cualitativo a los resultados del aprendizaje de sus alumnos como un criterio básico del diagnóstico integral? 
- ¿Reflexionan coligadamente maestros y alumnos para valorar resultados de la evaluación en un ambiente de aprendizaje socializado?

- ¿Propicia el maestro la auto evaluación, coevaluación y heteroevaluación en la clase?

- ¿Propician los maestros de la escuela primaria verdaderas situaciones de aprendizaje desarrollador para que el estudiante revele lo que ha aprendido?

- ¿Es considerada la evaluación como un proceso en el que se profundiza en los estilos y estrategias de aprendizaje en el aula y fuera de esta, o como un momento hostil y lleno de tensiones donde el alumno rinde cuenta de los resultados de determinadas tareas?

\section{DESARROLLO}

Son disímiles los criterios que con respecto a la evaluación se han valorado en los diferentes niveles de enseñanza, de forma general, maestros y profesores adoptan posiciones que van desde los extremos más conservadores, hasta las posiciones más tolerantes en cuanto a la determinación de la calidad de los resultados.

La evaluación es un componente del proceso de enseñanza-aprendizaje, forma parte de la dinámica que desde los inicios de cada actividad docente está determinada por la relación objetivo-contenido-método; no es un complemento ni elemento aislado. Cada maestro debe tener presente que lo más importante no es evaluar, ni hacerlo con la calidad requerida, lo más importante es saber cuáles son los propósitos en la dirección del proceso de enseñanza-aprendizaje, qué aspectos para el desarrollo de los alumnos son necesarios potenciar y en qué momento de la clase, curso o etapa es más propicio considerarlo para que se traduzca en aprendizaje.

Cuando en el contexto escolar hacemos referencia a la evaluación, generalmente pensamos en los alumnos, en qué aspectos del sistema de conocimientos es necesario profundizar, qué saberes han acumulado y cómo los exteriorizan, con la intención de comprobar cuál ha sido su rendimiento sin tener en cuenta otros factores que influyen considerablemente, ajenos a su voluntad, y que conducen a recrear situaciones fragmentadas, muy desvinculadas de la realidad, que alejan a la escuela del verdadero ideal social al que debe aspirar en la formación de las nuevas generaciones.

La evaluación en la escuela primaria se ha visto plagada de un cúmulo de insuficiencias, entre ellas podemos citar:

- La no consideración de un diagnóstico integral y acertado para conocer los antecedentes del aprendizaje de cada alumno.

- Son insuficientes las acciones de exploración de los conocimientos precedentes en la práctica pedagógica como criterio básico para determinar la zona de desarrollo actual y potencial con el propósito de precisar nuevas y superiores metas.

- Algunos maestros no son capaces de determinar niveles de logro en la adquisición de las habilidades como premisa para la selección de ejercicios con este fin.

- Existe una tendencia generalizada a centrar la atención en los resultados de las tareas sin atender las particularidades del proceso para lograrlo. 
- La evaluación no es concebida como parte del proceso de aprendizaje, sino como un momento de represión y rendición de cuentas de los resultados de los alumnos que lastran su independencia en este proceso.

- Falta de integralidad en el proceso evaluativo ya que no da respuesta a las exigencias para el aprendizaje del contenido.

- Las acciones del proceso de evaluación se centran en el maestro, por lo que no se incentiva la implicación del alumno en la determinación de la calidad de los resultados, el reconocimiento de su individualidad, su experiencia, niveles de ayuda que puede brindar a sus coetáneos y la autovaloración de los logros alcanzados.

- Como tendencia se manifiesta que el maestro evalúa más con la intención de clasificar un grupo de alumnos por el resultado, que por determinar las perspectivas de su desarrollo.

- No se aprovechan las dimensiones didácticas del error.

- Las dificultades en el aprendizaje se asocian más a problemas del alumno que a insuficiencias del maestro en su orientación y control.

- No se concibe a la evaluación como un proceso de seguimiento al diagnóstico inicial de los alumnos.

Estas insuficiencias, entre otras, nos revelan el divorcio que aún se manifiesta entre el aprendizaje y su evaluación, o sea, se fragmenta la integridad del proceso didáctico de la escuela primaria, el que tanto necesita el concurso de acciones que conduzcan a la reflexión de cómo transformarlo en un proceso más integral y desarrollador.

En reiteradas ocasiones, algunos detractores de la bilateralidad del proceso niegan la necesaria orientación que tienen que recibir los alumnos en sus diferentes estadios de desarrollo y cuando estos presenta determinadas insuficiencias en su aprendizaje culpan al maestro del nivel anterior, a la familia, o simplemente alegan que tienen problemas de aptitud para transitar por el grado o nivel.

Otros plantean que los únicos culpables del bajo rendimiento académico son los alumnos, sin hacer un análisis profundo de los antecedentes del aprendizaje de cada uno de ellos. No son pocos los maestros que erróneamente han remitido a un alumno con el especialista del centro de diagnóstico y orientación (CDO, institución cubana que atiende a los niños con dificultades en el aprendizaje y problemas conductuales), por no haber evaluado cualitativa y acertadamente los procederes individuales en la relación sistémica proceso-resultado-causa. En este sentido, cada maestro debe reflexionar sobre lo siguiente:

- ¿Están mis alumnos en igualdad de condiciones para aprender?

- ¿Cómo proceder para lograr la igualdad de oportunidades desde la propia clase?

Algunas consideraciones epistemológicas sobre la evaluación en la escuela primaria.

Todos los sujetos que aprenden y sobre todo, los de la edad escolar, están aptos para hacerlo, el maestro tiene que saber aprovechar dos cualidades básicas que son inherentes a esta etapa del desarrollo ontogenético y que distinguen el aprendizaje humano del de los animales: la inmadurez y la plasticidad: 
La primera, revela un estado de dependencia del sujeto por conocer, orientarse a la búsqueda de lo nuevo, preguntar constantemente, responder a cualquier estímulo de acuerdo con su variedad y novedad, y comparar sus resultados con los del otro; la inmadurez está muy asociada a la involuntariedad que caracteriza a los modos de actuación de estas edades; en el ámbito escolar, lo esencial por parte del maestro es orientar al alumno a que logre diferenciar qué actividades son las que más le aportan a su proceso de aprendizaje, que cada acción orientadora, desde la posición del alumno se convierta en la aprehensión de un método de aprendizaje y que los resultados de la evaluación le demuestren niveles de logros y exprese satisfacción por las transformaciones alcanzadas.

El criterio de plasticidad, como generalidad, desde la posición del maestro, ha estado muy asociado con el establecimiento de reglas o normas de trabajo colectivo o individual entre los alumnos de estas edades, al considerarlos seres indefensos y que siempre tienen que aceptar lo que se les orienta, sin que medie discusión o valoración alguna como condición básica para que se reconozcan actitudes positivas de acatamiento a lo que se dijo en un momento determinado.

Sin embrago, la generalidad de los maestros no ha sido capaz de combinar lo anterior con las posibilidades que brinda esta cualidad de las personalidades en formación, para iniciar, desde edades tempranas un profundo proceso formativo que comprometa al alumno con su autotransformación y que sienta la necesidad de transformar a los demás.

El maestro no debe dejar de considerar que en estas edades los alumnos sobredimensionan su influencia, que sus modos de actuación se convierten en un modelo que constantemente se multiplica, así, lo más importante no es imponerse ni declarar estilos que sean proclives a la hegemonía de la dirección unilateral, sino, lograr consenso entre todos, demostrarle al alumno que lo que diariamente se aprende va superando cualitativamente lo aprendido y que tienen amplias posibilidades para enfrentarlo: en ello se fundamenta la necesidad del cambio.

Así, "la inmadurez y la plasticidad del ser humano con respecto a las otras especies del reino animal determinan la importancia trascendental que tiene el aprendizaje para el desarrollo de los individuos maduros" (Dra D. Castellanos y otros, 2002.).

Tanto la inmadurez como la plasticidad son potenciadores y mediadores de un proceso de desarrollo, determinado en buena medida por la eficiencia de los procesos evaluativos a los que sea sometido el alumno para potenciar su aprendizaje y constatar, en cada etapa, fortalezas y debilidades de los resultados alcanzados y qué estrategia de intervención es la más adecuada para los nuevos propósitos, donde cualidad, valor e integración adquieren una preponderancia cada vez más sistémica.

Evaluar es atribuir valor a las cosas, es afirmar algo sobre su mérito. Se evalúa para comprender, alcanzar un cambio y mejorar. Es un instrumento que la escuela debe aprovechar al máximo, en la toma de decisiones, en la organización escolar y en la determinación de los índices de calidad en la formación de los alumnos.

La evaluación educativa es un fenómeno de extraordinaria complejidad en el cual se acumulan una serie de funciones diferenciadas e interrelacionadas, lque en la educación primaria adquieren una especial relevancia. Por una necesidad didáctica, estas funciones se separan para su estudio y puede que una adquiera mayor relevancia que otra en correspondencia con una determinada situación del proceso de enseñanza-aprendizaje, pero en la práctica educativa se manifiestan con carácter de sistema. Las 
ambiciosas finalidades de la evaluación la comprometen con un conjunto de funciones dirigidas a optimizar el proceso educativo en estas edades.

En la diversidad de fuentes consultadas no existe la distinción de un sistema de funciones de la evaluación para la escuela primaria, por lo que en las actuales condiciones del desarrollo de las ciencias sociales, especialmente la Pedagogía y la Psicología, han sido de obligado referente las tendencias de la pedagogía exsoviética y alemana contemporáneas, así como las particularidades de cada una de las etapas por las que ha transitado la Pedagogía en Cuba, según el Dr. C. Álvarez de Zayas; además de los criterios de L. Kilngber, (1974); M.A Danilov, (1978); G. Labarrere y G. Valdivia, (1988); Miguel S. Guerra, (1993); P. Maceda (1994); R. M. Álvarez, (1997); O. Castro (1998); M. Rivilla, (2000); J. Delval, (2001); F. Addine(2001) y MG. Ibar, (2002), entre otros.

De forma general, los postulados que se manejaban acerca de las funciones de la evaluación en la escuela cubana, hasta finales de la década de los 80 , estaban dirigidos a concebirla con un carácter procesual; pero muy ligada al desempeño del maestro desde su posición de dirigente del proceso de enseñanza-aprendizaje. Eran entendidas como funciones que hacía cumplir el maestro, más bien para transmitir conocimientos que para propiciar el desarrollo de los alumnos.

Estaba muy arraigada la tendencia de concebir a la evaluación como un instrumento para constatar el proceso de apropiación de los conocimientos de los alumnos, además prevalecía el criterio de que los resultados del aprendizaje estaban en dependencia de la cantidad de evaluaciones que el alumno acumulara en formas de juicios de valor, sin que mediara análisis cualitativo alguno del desarrollo de las habilidades en el proceso y mucho menos de indicadores que lo determinaran en cada una de las etapas de su desarrollo ontogenético.

En la escuela primaria se manifiesta como tendencia (por la preponderancia del autoritarismo arraigado por la fuerza de la tradición) la no-consideración de los criterios de los que aprenden con respecto a lo que cada uno necesita, los alumnos no son orientados hacia la autovaloración y cómo valorar al otro; ni se les enseña a diferenciar cuáles son sus necesidades de ayuda y cómo la deben brindar a los demás.

Por otra parte, desde nivel central (MINED) eran propuestos sistemas de evaluación muy rígidos y descontextualizados, lo que conducía a que la mayoría de los maestros (para no absolutizar el defecto) centrara su atención en el resultado cuantitativo que determinaba el avance por cada etapa del curso o semestre. La escuela primaria estaba fragmentada: existían maestros especialistas de un primer y segundo ciclo; como tendencia, el tránsito del alumno de un grado a otro se hacía con algunas insuficiencias que el maestro emisor nunca detectaba y era culpado por el del grado posterior: en suma no se concebía la importancia de los ciclos como momentos del desarrollo sino como formas estereotipadas de organizar la estructura de la escuela.

La participación del alumno en el proceso de evaluación era concebida únicamente a partir de cinco funciones (instructiva, educativa, de diagnóstico, de desarrollo y de control) con un marcado carácter animista, o sea, el hecho de que el maestro conozca teóricamente la función era condición básica para que esta se cumpliera en el proceso, sin considerar la diversidad de formas de evaluación que puede implementar el maestro a partir de la implicación consciente del alumno, de sus peculiaridades personales e intelectuales. 
Independientemente de los logros alcanzados por maestros y alumnos en las diferentes clases a partir de las actuales transformaciones, todavía se manifiestan en la evaluación procederes manidos, tendentes a lo cuantitativo, para la determinación de los conocimientos que van acumulando los alumnos, sin registrar las particularidades del tránsito por el proceso que lo condujo a un determinado resultado.

La experiencia precedente, de más de veinte años en la formación de profesionales para la escuela primaria, ha demostrado que desde el propio Instituto Superior Pedagógico, donde prevalecen las estrategias didácticas del enseñar a aprender y enseñar a enseñar, no se ha logrado que estos estudiantes (tanto los del curso diurno como los del curso para trabajadores) logren dominar plenamente cuáles son sus habilidades profesionales, qué acciones las sustentan, en qué etapas del curso o semestre deben alcanzarse y qué indicadores evidencian que los modos de actuación se van transformando de acuerdo con las exigencias del modelo del profesional; por lo que es una insuficiencia que por transferencia también se ha manifestado en el aprendizaje de los alumnos de la escuela primaria.

Esto quiere decir que los alumnos de la escuela primaria no siempre están lo suficientemente orientados a identificar logros y dificultades de su aprendizaje a partir de los resultados del diagnóstico y en correspondencia con ello, determinar de forma perspectiva qué estrategia de aprendizaje adoptar dentro o fuera del aula para hacer más eficiente su autoaprendizaje.

Es por ello que el trabajo del maestro debe estar dirigido a que el alumno interiorice conscientemente qué es lo que debe aprender, por qué le necesita, que reconocimiento social recibirá de sus coetáneos, familiares y maestros y que se sientan estimulados con la ejecución de cada tarea en el proceso de aprendizaje.

La concepción de evaluación que como regularidad prevalece en la escuela primaria actual, es una manifestación de la concepción de aprendizaje, centrado básicamente, en la figura del maestro, en su autoridad, determinada en buena medida por la desconfianza hacia el autoaprendizaje e independencia de los alumnos y por el temor de que estos lo hagan quedar mal ante los directivos que en diferentes momentos del curso controlan su trabajo.

Se manifiesta como una regularidad de la escuela primaria actual que el maestro sobredimensione lo instructivo con respecto a lo educativo, por lo que las acciones para constatar la evolución de los alumnos, se reducen en buena medida, a comprobar cúmulo de conocimientos y no las posibilidades que tienen de utilizarlos en la vida cotidiana para su beneficio propio o el de los demás.

Así, el tránsito del alumno por las diferentes etapas del curso está avalada, generalmente, por un resultado cuantitativo que se expresa en un juicio de valor y que nada aporta al proceso de diagnóstico y caracterización tan necesarios en estas edades.

Otro aspecto a considerar es que en el actual proceso de transformación y perfeccionamiento por el que transita la escuela cubana la ubica a la vanguardia de la educación en América Latina y el Caribe, donde se destaca la reducción de la relación alumno-maestro en cifras de hasta 20 alumnos en cada aula

En este proceso de constante perfeccionamiento, se crean un conjunto de condiciones que favorecen extraordinariamente la realización del proceso docente-educativo hacia un estadio de desarrollo mucho más cualitativo, esto hace posible que la problemática que se aborda manifieste una tendencia 
positiva en el tránsito hacia etapas superiores, no obstante, estas condiciones no son lo suficientemente aprovechadas por todos los maestros para que a través de la evaluación se logre el impacto social de las transformaciones deseadas en la adquisición de conocimientos por cada alumno; por lo que cabe reflexionar:

¿Es posible mantener una evaluación cuantitativa, sumativa y no participativa en aulas de hasta 20 alumnos?

No enfrentar los retos del cambio ubicaría a los maestros en el centro de censura de sus alumnos, directivos, familiares y la sociedad en general, la propia dinámica del proceso los irá comprometiendo, cada vez más, con el desempeño de sus funciones.

Las condiciones antes referidas y las tendencias a la mejoría en este sentido, en modo alguno, nos liberan de continuar profundizando en las particularidades del proceso de evaluación para la escuela primaria.

Son pocos los autores que valoran la importancia de la evaluación con la perspectiva de hacer más eficiente el aprendizaje, generalmente ha sido concebida como el instrumento de comprobar la apropiación de los conocimientos por parte de los alumnos, como el espacio para la rendición de cuentas de lo que se ha aprendido, para llenarlos de tensiones en diferentes etapas del curso escolar.

Otro de los aspectos que ha sido pobremente tratado con respecto a la relación evaluaciónaprendizaje, es lo relacionado con la consideración de las dimensiones didácticas del error. (S. De la Torre, 1999. Aprender de los errores y M. Velázquez, 2002. La dimensión didáctica del error).

Está demostrado que en la dirección del aprendizaje la generalidad de los maestros considera el error con una connotación negativa, de derrota, tendente a clasificar al grupo, a crear barreras en el aprendizaje, y a transmitirle al alumno el fatídico sentimiento de que es un fracasado. Sin embargo, el error debe concebirse como un momento más por el que transcurre el aprendizaje; lo más importante es que el maestro enseñe al alumno a reorientarse didácticamente en el proceso a partir de los errores, que estos no deben asociarse únicamente con la evaluación y que son decisivos, a favor del aprendizaje, en cualquier momento del proceso.

Por lo anteriormente expuesto y por la importancia de hacer más integradora la evaluación, proponemos un sistema de funciones dirigidas a optimizar este proceso en la escuela primaria; de las nueve funciones que proponemos seis ya habían sido tratadas por diferentes autores cubanos y para otros niveles de enseñanza: las tres últimas (función participativa, metacognitiva y ecológica) las sometemos a su consideración como un modesto aporte al mejoramiento de este componente de la Didáctica que merece especial atención para lograr un aprendizaje consciente y desarrollador de los alumnos de la escuela primaria.

\section{FUNCIÓN DE DIAGNÓSTICO}

Permite saber cuál es el estado cognoscitivo y actitudinal de los alumno; así ajustar la acción de intervención a sus características, peculiaridades, saberes, intereses, motivaciones y expectativas. Un diagnóstico acertado facilita un aprendizaje significativo y relevante ya que parte del estado real (problema) la situación precedente y las causas que la sustentan. Es importante diagnosticar contexto y las condiciones 
en que se desarrollará el proceso, anticipar de alguna manera, qué se espera de cada sujeto, qué ayuda recibe del otro y del propio maestro, enseñarlo a demandar la ayuda para que pueda comprender cuál es su situación real de aprendizaje.

\section{FUNCIÓN SELECTIVA}

Esta función, lejos de definir una determinada posición del sujeto en el grupo y predeterminar un futuro social, (Santos Guerra, 1993) que es un criterio incompatible con nuestro modelo educativo, se implementa, en tanto necesidad didáctica para el maestro, en definir qué contenido va a reforzar, qué medios audiovisuales son necesarios para el aprendizaje, los alumnos con los que el maestro formará un equipo, trabajos realizados para ser exhibidos, locales y horarios adecuados, de alumnos para que dirijan una actividad y expongan sus conocimientos y experiencias, entre otros. Con esta función se debe hacer ver al alumno que la evaluación propicia la toma de decisiones, por ejemplo, en cuanto a qué se estudia, con quién lo hace, qué fuente de información utiliza. En suma, que la selección lo aproxime a ser cada vez más independiente.

\section{FUNCIÓN DESARROLLADORA}

Es un arma eficaz para valorar el trabajo realizado por el maestro, y someterlo a una revisión y actualización permanente, como necesidad de una constante superación científico -pedagógica.

En la medida en que defina acertadamente los problemas, las causas y su influencia en el aprendizaje de sus alumnos, el nivel de los logros en los resultados que corroboren la efectividad de las acciones seleccionadas por él, serán resortes motivacionales de dirección y resultados de maestros y alumnos respectivamente. Situaciones de aprendizaje más desarrolladoras revelarán más acertadamente el aprendizaje de los alumnos.

\section{FUNCIÓN DE JERARQUIZACIÓN}

Esta función permite organizar de forma gradual la atención a los alumnos con dificultades en determinados aspectos del conocimiento o habilidades, los que tienen problemas de puntualidad, con la expresión oral, cumplimiento con la presentación formal de algún trabajo.

Aunque no exista una marcada intención de diferenciar por parte del maestro, la jerarquización, involuntariamente marca niveles de diferencia entre los alumnos, lo más evidente está relacionado con la definición del aprobado o desaprobado, determinar los niveles de ayuda, la prioridad de atención que merece cada sujeto, qué contenidos de los tratados nos aproximan más a los propósitos mediatos e inmediatos de la dirección del aprendizaje, qué estrategia evaluativa, seleccionar y en qué orden se introducen las acciones para la autoevaluación y coevaluación.

Los maestros deben cuidar que esta función no se convierta en un instrumento humillante o de segregación del colectivo, en la jerarquización, como característica inherente a los sistemas (en este caso sujetos del proceso) deben interactuar todos sus integrantes, maestros y alumnos sin distinción. Son jerarquías necesarias para el mejoramiento del proceso docente. (RM. Álvarez, 1997).

\section{FUNCIÓN DE COMUNICACIÓN}


La interrelación entre los sujetos que intervienen en el proceso docente educativo es decisiva para alcanzar los resultados, valorarlos e interpretarlos. La comunicación tiene connotadas repercusiones psicológicas para el alumno y el maestro (MA. Santos G. 1993). El alumno en este proceso ve la posibilidad de autoafirmarse y elevar su autoestima dentro del colectivo, de compartir acuerdos y reflexionar sobre las disquisiciones para llegar a consensos entre los demás miembros del colectivo, oír las consideraciones del otro, ayudarlo a través del diálogo, sin imposición, que cada sujeto sienta que sus criterios tienen significado para la mayoría o una buena parte del grupo.

Es importante que la receptividad como interlocutores sea perceptible visualmente, aspecto este decisivo para la necesaria empatía entre evaluadores y evaluados; que desde la primera impresión sea un diálogo constructivo, ameno, enriquecedor, de apertura, sin reservas, donde se exprese con sinceridad lo que cada alumno ha aprendido, cuál ha sido su protagonismo y si encierra la verdadera dimensión ética que presupone este acto.

\section{FUNCIÓN DE ORIENTACIÓN}

La orientación es un componente básico de la actividad de aprendizaje, es un aspecto potenciador de la esfera inductora motivacional de la personalidad. Después de registrados los resultados, se valoran los logros, dificultades y causas asociadas como criterios básicos para reorientar el trabajo que hasta ahora se había estado realizando.

Presupone reflexionar sobre ¿cuáles fueron los problemas?, ¿Qué hacer?, ¿Qué cambiar?, ¿Qué dirección tomar? ¿Cuál es la más acertada de todas las alternativas? . La orientación es el momento de reconsiderar, cambiar, de empezar un proceso cualitativamente superior. (RM Álvarez, 1997). Es el momento propicio para promover la importancia de las expectativas, estimular la adopción de normas y reglas para todas las actividades que se desarrollan en la escuela como fundamento de un proyecto de vida más mediato.

\section{FUNCIÓN PARTICIPATIVA}

El carácter social y participativo de la evaluación la comprometen cada vez más con la implicación y protagonismo del alumno en el proceso y de sus responsabilidades como sujeto de aprendizaje. Se acrecienta el compromiso social de intervenir para mejorar el proceso, brindar la ayuda que necesitan los demás y que cada uno declare lo que necesita en este sentido. La consideración de los niveles de ayuda es decisiva para la implicación de cada alumno en el proceso; es por ello que su participación consciente, reflexiva, libre de prejuicios, permiten que el aprendizaje revele su verdadero carácter social e interactivo.

\section{FUNCIÓN METACOGNITIVA}

La implicación consciente del alumno en el proceso de enseñanza-aprendizaje y la constatación de su evolución para llegar a un resultado a partir de la evaluación, demandan de una actitud más autorefelxiva de los logros de su aprendizaje, que en cada etapa de la evaluación el maestro lleve al alumno a profundizar en sus propios procesos cognitivos, a regularlos, a planificar sus tareas y a diferenciar la inmediatez de la mediatez, a tolerar posiciones y estadios de desarrollo de las demás personas, a que se evidencie en los momentos de la actividad de aprendizaje la relación entre el saber qué y el saber cómo. 
Posibilita potenciar las relaciones afectivas, los vínculos contexto-autorregulación, el autodiagnóstico, la supervisión y la cooperación.

\section{FUNCIÓN ECOLÓGICA}

La evaluación del proceso de enseñanza-aprendizaje se desarrolla muy vinculada a la vida del sujeto que aprende, el cual está inmerso en un medio sociocultural complejo y contradictorio que se caracteriza por las relaciones sociales recíprocas de dar y recibir, en muchos casos, con una considerable dosis de involuntariedad, descentralización y dejar hacer que sin llegar a la anarquía y desobediencia si proliferan modos de actuación que no se corresponden con las más elementales normas de convivencia, sobre todo, cuando la escuela no interviene oportunamente para contrarrestar estas inadecuaciones sociales.

Es por ello que en la dirección del proceso de enseñanza-aprendizaje, desde edades tempranas el maestro tiene que trabajar para lograr entre los sujetos recurrentes relaciones que se caractericen por la espontaneidad, despojadas del lastre de la subjetividad y la suposición, siempre con la buena intención de ayudar al otro, que cada uno se libere de prejuicios y tabúes para dar criterios de los resultados individuales o grupales.

Rechazar puntos de vistas o posiciones y no personas, aceptar el trabajo en grupos independientemente de su composición racial y social, lo que influye considerablemente en la determinación del impacto humano sobre el entorno social cuando todo ello está en función de elevar la calidad de vida.

Por la complejidad del proceso docente-educativo en la escuela primaria, y por la necesidad del cambio que ello requiere, relaciono una serie de aspectos que el maestro no puede dejar de considerar para evaluar el aprendizaje de sus alumnos:

1. Partir de un diagnóstico integral y acertado, lo que posibilitará:

- Remitirse a las particularidades de los precedentes en el proceso de asimilación de los conocimientos.

- Determinar hacia dónde se orientan las fortalezas, dónde radican las insuficiencias en la apropiación de habilidades y métodos de aprendizaje.

- Establecer niveles de ayuda maestro-alumno, alumno-alumno, maestro-familia y familiaalumno.

- Caracterización profunda de la familia con énfasis en las potencialidades de esta para darle continuidad al proceso de aprendizaje en el hogar.

- Determinar antecedentes patológicos personales y familiares que intervienen en el aprendizaje.

2. Propiciar que el alumno establezca nexos entre lo conocido y lo nuevo por conocer

3. Utilizar preguntas dirigidas a la reflexión y que orienten al alumno hacia el análisis de las condiciones en que se ejecuta la tarea.

4. Analizar las posibilidades de diferentes vías de ejecución de ejercicios y tareas.

5. Propiciar la ejecución de tareas en diferentes niveles de interacción. 
6. Potenciar procesos de autoevaluación del alumno.

7. Diferenciar la calificación de los procesos de aprendizaje que la evaluación propicia.

8. Someter su estilo de evaluación a la consideración de otros maestros.

9. Oír el parecer de sus exalumnos sobre la forma de evaluar.

10. Intercambiar criterios con otros maestros sobre resultados evaluativos de sus alumnos.

Para lograr mayor diferenciación en el proceso de evaluación de los alumnos del primer y segundo momentos del desarrollo (preescolar a segundo grado) se deben considerar los siguientes aspectos:

- Partir de un diagnóstico integral y diferenciado de los antecedentes del alumno.

- Diferenciar logros y dificultades en el aprendizaje de los procesos de lectura y escritura, operaciones elementales de cálculo y de nociones primarias sobre la naturaleza y sociedad. El control y seguimiento de estos aspectos del contenido, le permitirá al maestro concebir a la evaluación como un proceso que permite el aprendizaje.

- Atender y estimular diferenciadamente el desarrollo del carácter voluntario y consciente que gradualmente adquieren los procesos psíquicos en estas edades.

- A través de ejercicios y tareas, potenciar el desarrollo de los procesos lógicos del pensamiento como el análisis y la síntesis, establecimiento de relaciones, entre otros, como aspectos esenciales para diferentes aprendizajes como la lectura, la escritura y la matemática.

- Orientar tareas donde se propicie la utilización de medios auxiliares, para que puedan memorizar, estableciendo relaciones para promover una retención lógica y no mecánica.

- Ofrecer tareas de aprendizaje que despierten el interés del niño hacia los temas que desarrolla en clases y asuma actitudes conscientes hacia los conocimientos que adquieren.

- Orientar ejercicios, tareas individuales y colectivas dirigidas a que el alumno trabaje con materiales que sean directamente perceptibles mediante modelos adecuados.

\section{CONCLUSIONES}

La complejidad y riqueza del proceso docente-educativo irradia a la evaluación este mismo carácter, por lo que constantemente es susceptible de perfección para hacer más eficiente la labor de enseñanza y aprendizaje.

Profundizar sistemáticamente en la implementación de estas funciones nos lleva a reflexionar sobre los matices 'personológicos' del aprendizaje escolar. ¿Qué nuevas experiencias deben registrarse por los maestros en ejercicio para reorientar el trabajo hacia la evaluación en todas sus modalidades, implicar y comprometer conscientemente a los alumnos con los resultados individuales y grupales, que a partir de las acciones de autovaloración y valoración del otro se eliminen las asperezas que puedan surgir en este proceso, persuadir a los de menos posibilidades de aprendizaje de cuáles son las causas de sus resultados y estimular a los que han alcanzado más calidad para que continúen evolucionando hacia niveles de excelencia?

La concepción sistémica de las funciones de la evaluación y las acciones que de ella se derivan la hacen acentuar su omnipresencia en el proceso formativo de la escuela primaria, por lo que maestros y directivos deben trabajar por lograr su extensión a la familia y a la microsociedad del alumno, donde debe 
prevalecer la naturalidad, voluntariedad, independencia y cotidianidad; sin buscar momentos o encuentros especiales para constatar qué ha aprendido el alumno, o qué niveles de ayuda necesita.

Evaluar en la escuela primaria no es en modo alguno, clasificar grupos o alumnos de acuerdo con sus posibilidades, ni dar una calificación para buscar una relación proporcional entre el juicio de valor y los conocimientos adquiridos: todo aprendizaje está avalado por resultados cualitativos que van evolucionando a través de diferentes momentos del desarrollo, en la dinámica de la interacción con los demás sujetos, en la comprensión más profunda de los resultados que alcanza y en sus causas para orientarse perspectivamente hacia estadios más cualitativos de autoaprendizaje.

\section{BIBLIOGRAFÍA}

AdDINE F, Fátima (1998): Didáctica y optimización del proceso de enseñanza - aprendizaje. La Habana: IPLAC. Pueblo y Educación. Junio, 1998.

ÁlvAREZ DE ZAYAS, Carlos M. (1993): La escuela en la vida. La Habana: Editorial Félix Varela. 1993.

(1998): Pedagogía como ciencia o Epistemología de la educación. La Habana: Félix Varela. 1998.

ÁlvAREZ DE ZAYAS, Rita Marina (1990): El desarrollo de las habilidades en la enseñanza de la Historia. La Habana: Pueblo y Educación. 1998.

(1997): Hacia un curriculum integral y contextualizado. Tegucigalpa: Ed. Universitaria. 1997.

DE LA TORRE, Saturnino (1993): Aprender de los errores. Madrid. Editorial Escuela Española. 1993.

DELORS, Jacques (1996): La educación encierra un tesoro. Madrid: Santillana, Ediciones UNESCO. 1996.

DelVAL, Juan (1990): Lo fines de la educación. Ediciones Siglo XXI. Madrid-México. 1990.

DeLVAL, Juan (2001): Aprender en la vida y en la escuela. Madrid: Morata. 2001.

Delval, Juan "Tesis sobre el Constructivismo". En: La construcción del conocimiento escolar, Paidós Barcelona pp 15-33. Año 1997.

García Ramis, Lisardo. (1996): Los retos del cambio educativo. La Habana: Pueblo y Educación. 1996.

IBAR, G. Mariano (2002): Manual general de evaluación. Madrid. España. Ediciones Octaedro. 2002.

MedinA RIVILLA, Antonio "La actividad, base del proceso de enseñanza-aprendizaje en el aula " En: La calidad en los procesos educativos, Oikos-Tau. Barcelona. pp 205-236. Año 2000.

RIco, Pilar y otros (2002): Hacia el perfeccionamiento de la escuela primaria. La Habana. Editorial Pueblo y Educación. 2002.

SANTOS GUERRA, Miguel (1993): La evaluación: un proceso de diálogo, comprensión y mejora. Málaga Granada, Ediciones Aljibe, 1993.

VeLÁZQUEZ TEJEDA, Miriam (2001): La dimensión didáctica del error en la enseñanza de la Lengua Española. Sitio web monografías.com. 